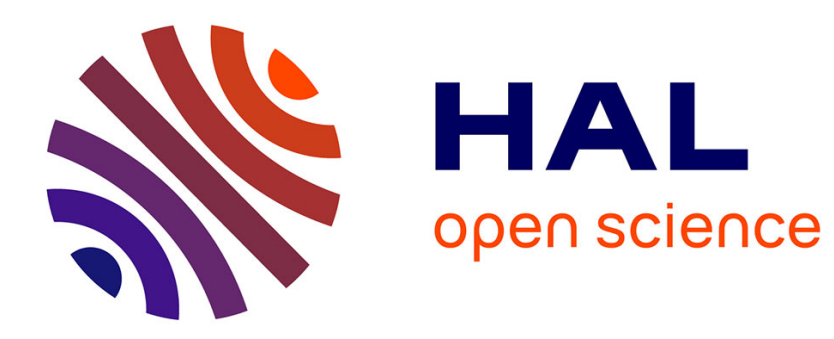

\title{
Aspect mécanique de la croissance des racines. I. - Mesure de la force de pénétration
}

\author{
Nicole Souty, Colette Rode
}

\section{To cite this version:}

Nicole Souty, Colette Rode. Aspect mécanique de la croissance des racines. I. - Mesure de la force de pénétration. Agronomie, 1987, 7 (8), pp.623-630. hal-00885035

\section{HAL Id: hal-00885035 \\ https://hal.science/hal-00885035}

Submitted on 1 Jan 1987

HAL is a multi-disciplinary open access archive for the deposit and dissemination of scientific research documents, whether they are published or not. The documents may come from teaching and research institutions in France or abroad, or from public or private research centers.
L'archive ouverte pluridisciplinaire HAL, est destinée au dépôt et à la diffusion de documents scientifiques de niveau recherche, publiés ou non, émanant des établissements d'enseignement et de recherche français ou étrangers, des laboratoires publics ou privés. 


\title{
Aspect mécanique de la croissance des racines. I. - Mesure de la force de pénétration
}

\author{
Nicole SOUTY \\ avec la collaboration technique de Colette RoDE \\ I.N.R.A., Station de Science du Sol, B.P. 91, Centre de Recherches d'Avignon, F 84140 Montfavet
}

RÉSUMÉ

\begin{abstract}
Dans un sol, milieu hétérogène, une racine peut rencontrer des zones de résistance différente à la pénétration qui font obstacle à son enfoncement et à son développement. En laboratoire, nous avons modélisé un tel obstacle par une lamelle d'acier sur laquelle est collée une jauge extensométrique. Nous pouvons ainsi déterminer simultanément la force et la vitesse de croissance de la racine ; de plus, nous suivons l'évolution des structures cellulaires méristématiques en observant des coupes cytologiques. Nous comparons cette méthode à d'autres présentées dans une revue bibliographique.
\end{abstract}

Mots clés additionnels : Propriétés mécaniques, vitesse, cytologie.

\begin{abstract}
Mechanical behaviour of growing roots. I. - Measurement of penetration force.
The influence of soil physical properties on the growth and morphology of the maize seedling root was studied in the laboratory. An experimental method was developed to determine growth force and growth rate : the root extends against an obstacle which is made of a small plate of steel attached to a strain gauge. This method is compared with others in a bibliographical study.
\end{abstract}

Additional key words : Mechanical properties, rate, cytology.

\section{INTRODUCTION}

Bien qu'elles n'interviennent pas seules, mais en interaction avec les autres caractéristiques du milieu physique, les propriétés mécaniques du sol et leur variabilité apparaissent fréquemment déterminantes pour le développement et la croissance du système racinaire d'une culture au champ (BARLEY \& GREACEN, 1967 ; TAYLOR \& BURNETT, 1964 ; STOLZY \& BARLEY, 1968).

La connaissance des relations entre les propriétés mécaniques du sol et la croissance racinaire est donc un élément nécessaire à la prévision de la distribution spatiale du système racinaire et de sa dynamique, ellemême indispensable à la modélisation de l'absorption d'eau et de solutés par les plantes (TARDIEU \& MANICHON, 1986).

L'étude analytique de ces relations ne peut, de façon réaliste, se donner pour objectif l'élaboration d'un modèle général et déterministe de calcul de la morphologie de la racine à partir de données physiques décrivant le sol. En revanche, la connaissance des lois élémentaires du comportement mécanique d'une racine en croissance peut permettre d'envisager la conception de modèles probabilistes dépassant le stade actuel de la corrélation avec les observations de la structure du sol, la densité ou la résistance à la pénétration. C'est la démarche suivie depuis quelques années par DEXTER (1978) ; elle suppose que l'on étudie dans un premier temps, par voie expérimentale, les forces exercées par une racine placée dans des milieux de propriétés mécaniques contrôlées et variées, ces déterminations étant complétées par des mesures de vitesse et des observations de déformations.

Du fait de la difficulté des mesures in situ dans un matériau tel que le sol, on est alors conduit à étudier une racine placée en contact avec des obstacles artificiels, mécaniquement bien connus. La réalisation d'un tel dispositif, la relation des informations fournies, avec le comportement mécanique de cette racine en croissance se heurtent à de nombreuses difficultés, à en juger par les travaux d'autres auteurs.

Après une analyse bibliographique des techniques existant et des résultats acquis, nous présentons dans cet article l'originalité de notre méthode et ses domaines d'application. Dans un article suivant, nous ras- 
semblerons les résultats concernant l'influence des propriétés mécaniques de l'obstacle sur la force axiale et la vitesse de croissance de la racine.

\section{II. ÉTUDE BIBLIOGRAPHIQUE}

\section{A. Observations générales}

L'extrême variabilité des propriétés du milieu, souvent interdépendantes, agit sur le développement et la croissance racinaire et par là même sur les interactions de la racine avec le milieu.

En effet des variations de l'état physique du sol affectent non seulement la résistance mécanique, mais aussi l'aération (GILL \& MILlER, 1956 ; PERIGAUD, 1966) et les flux d'eau et d'éléments nutritifs (PHILLIPS \& DOM KIRKHAM, 1962 ; GARDNER, 1964 ; NYE \& MARRIOTT, 1969 ; DREW, 1975).

Les observations d'une racine dans un milieu naturel mettent en évidence soit un changement de direction de celle-ci sous l'action d'une contrainte mécanique axiale, soit des déformations et des modifications du sol dues à la croissance de la racine (BARLEY \& GREACEN, 1967). Cependant, la plupart des chercheurs se sont intéressés uniquement à la racine : leurs essais (en pots essentiellement) dans des carottes de sol sont relatifs à la croissance racinaire et prennent en compte les paramètres d'une part du végétal, d'autre part du sol en début d'essai (c'est-à-dire : teneur en eau, potentiel, densité, lissage de la surface...). Certains se sont limités à des mesures de longueur de racines dans le temps (donc des vitesses d'allongement), (BARLEY, 1962; REICOSKY et al., 1970 ; BATCHELDER \& BOULDIN, 1972), d'autres ont compté le nombre de racines pénétrant dans l'échantillon de sol (BOEHLE et al., 1963 ; MELHUISH, 1968 ; PreBbLE, 1970). Enfin, plus récemment, des chercheurs tels que NGUYEN (1977), DEXTER \& HewitT (1978), DEXTER (1978), Whiteley et al. (1981), s'intéressent à la force, à la vitesse de pénétration et au cheminement des racines rencontrant soit un obstacle, soit un pore.

De façon générale, dans tous ces travaux, le sol est caractérisé, outre par le potentiel hydrique et la densité sèche à l'humidité considérée, par la résistance à la pénétration mesurée à l'aide d'un pénétromètre qui donne des valeurs toujours supérieures aux forces développées par les racines (NGUYEN, 1977 ; WHITELEY et al., 1981). Ces écarts proviennent des comportements très différents de la racine et du pénétromètre. En effet, la racine est capable de changer de forme et de grandir dans une direction de moindre effort, elle secrète un mucigel jouant le rôle de lubrifiant, par absorption d'eau, elle peut créer des fissures dans le sol environnant, enfin, la vitesse d'enfoncement de la racine $(1$ à $2 \mathrm{~cm} / \mathrm{j})$ est beaucoup plus faible que celle de l'aiguille du pénétromètre ( $3 \mathrm{~mm} / \mathrm{mn}$ environ) habituellement utilisé sur le terrain (BARLEY \& GREACEN, 1967 ; EAVIS, 1967 ; EAVIS et al., 1969 ; GOODERHAM, 1973 ; GOSS, 1974 ; RUSSELL \& GoSs, 1974).

Précédemment, nous avons signalé les travaux relatifs à la détermination de la force et de la vitesse, mais il ne faut pas négliger les résultats concernant le mode de cheminement d'une racine : en effet, une racine atteignant dans un sol l'interface de deux milieux peut soit pénétrer le deuxième milieu, soit être déviée. DEX TER (1978) a proposé un modèle mathématique de croissance en tenant compte à la fois de la résistance à la pénétration du deuxième milieu et de l'angle fait par la racine avec le plan tangent à la surface de ce milieu. Il peut arriver aussi que la traversée de l'interface soit limitée par le flambage de la racine si elle rencontre un pore dans lequel elle n'est plus frettée (WHITELEY et al., 1982). En effet, la racine présentant une attache fixe (vers la plantule) et une extrémité mobile (apex) pouvant fonctionner comme une rotule, peut, sous l'action d'une compression exercée à l'apex, prendre une courbure : c'est le flambage. L'entourage de la racine par un matériau rigide (mise en place d'une frette) permet d'éviter ce phénomène.

Par ailleurs, la présence d'une fissure entre les milieux (WHITELEY \& DEXTER, 1983) modifie le nombre de racines pouvant pénétrer le deuxième milieu : ce nombre diminue avec l'augmentation de la largeur de la fissure. En conditions identiques, les racines les plus fines pénètrent en moins grand nombre que les racines épaisses. Dans la fissure, le diamètre de la racine est à la fois fonction de la largeur de la fissure et de la résistance du sol; les auteurs ont d'ailleurs proposé une équation empirique pour prendre en compte ces effets.

Ces travaux mettent en évidence les difficultés à réaliser des mesures de pression racinaire, même lorsqu'il ne s'agit que d'une racine isolée. Néanmoins, nous pouvons supposer qu'en laboratoire, où nous sommes capables de réaliser des milieux continus, homogènes, isotropes et où les conditions hydriques et nutritives peuvent être maîtrisées, des mesures de force et de vitesse de croissance peuvent être envisagées. De plus, les propriétés mécaniques du milieu d'accueil peuvent régir essentiellement cette croissance si tous les autres paramètres sont fixés. Dans ces conditions, nous pensons que la détermination de la force développée par la racine et de l'allongement correspondant, sont en relation avec une évolution des structures végétales de la racine. Aussi, l'analyse de la morphologie cellulaire de la région apicale de la racine pourrait être un test intéressant dans la mesure où il pourrait être corrélé avec les paramètres mécaniques de l'environnement.

\section{B. Mesure de la force de croissance}

Depuis 1893, année où PFEFFER a entrepris les premières mesures de forces axiales (et radiales) de racines, de nombreux auteurs se sont penchés sur ce problème.

GILL \& BOLT (1955), TAYLOR \& RATLIFF (1969), EAvis et al. (1969) se sont inspirés de ces expériences en modifiant plus ou moins le dispositif de mesures de PFEFFER (fig. 1). La partie de la racine délimitee par les deux blocs de plâtre est située à des distances variables de l'apex ( 0 à $6 \mathrm{~mm}$ ) et les pressions axiales calculées à partir de la force et du diamètre de la partie libre de la racine sont de l'ordre de 6 à 25 bar alors que les pressions radiales sont inférieures à 6 bar. Le fait de ne prendre en compte que la croissance d'une zone étroite $(\leqslant 1 \mathrm{~mm})$ de la racine est certainement critiquable : il a été montré, en effet, en physiologie, que la zone d'élongation atteint à peine $1 \mathrm{~cm}$ de longueur en arrière de la coiffe, que le maximum d'élongation a lieu dans les 5 premiers mm pour diminuer au-delà. De plus, l'atmosphère à l'intérieur des blocs de plâtre n'est 


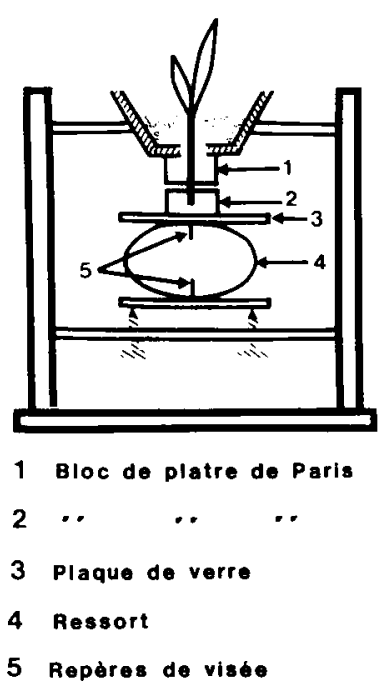

Figure 1

Dispositif de Pfeffer pour la mesure de la pression axiale de croissance.

Pfeffer's apparatus for measuring axial root pressure.

ni définie, ni contrôlée : il peut y avoir un problème d'aération et une influence sur la pression osmotique. Enfin, la valeur du diamètre utilisée pour le calcul de la pression ne doit certainement pas être celle du diamètre initial par suite, vraisemblablement, d'un élargissement de la zone non confinée dans le plâtre.

Au lieu de déterminer la pression propre à la racine, d'autres auteurs ont abordé le problème différemment : une pression externe est exercée sur la racine et la valeur pour laquelle apparaissent des modifications de la forme et de la vitesse d'élongation, est notée. Cette pression limite que peut supporter la racine est assimilée à sa pression maximale de croissance (GILL \& MILlER, 1956 ; AUBERTIN \& KARDOS, 1965 ; ABDALLA et al., 1969 ; Goss, 1974). Ainsi, des graines de blé mises à germer sur un lit de billes de verre (de $50 \mu \mathrm{m}$ de diamètre) pris entre une plaque rigide et un diaphragme en caoutchouc, sont soumises, par admission de gaz à des pressions pouvant atteindre 10 bar (GILl \& Miller, 1956). Sous 1,5 bar de pression, les racines séminales s'aplatissent, deviennent noueuses et anormalement ramifiées : leur vitesse d'élongation est de $1,3 \mathrm{~mm} / \mathrm{h}$ environ et passe à $0,15 \mathrm{~mm} / \mathrm{h}$ sous 2,7 bar.

En 1969, ABDAlla et al. à partir d'expériences sur orge ont proposé une explication mécanique de la pénétration du milieu par une racine. Dans un appareil triaxial modifié et rempli de billes de verre (ou de sable ou d'argile), une plantule d'orge est soumise à des pressions hydrostatiques de 1 bar au maximum. A partir de 0,2 bar, les auteurs constatent un léger effet sur la croissance racinaire, traduit par un épaississement, si par ailleurs les facteurs physiologiques et chimiques de croissance sont favorables. Par contre, ils remarquent une atrophie complète de la racine sous une pression de 0,6 à 0,7 bar. Ils suggèrent alors, dans le domaine de pressions considéré, l'existence de $3 \mathrm{com}$ portements successifs de la racine : allongement axial normal, début d'épaississement et enfin arrêt total de toute croissance. Une racine étant cependant capable de pénétrer un milieu sol résistant, ces auteurs pensent que, dès que la pression exercée est suffisante pour inhiber l'allongement axial, la région méristématique s'élargit. Ce mécanisme d'épaississement s'observe d'ailleurs fréquemment dans les milieux à fortes contraintes mécaniques, dans les couches profondes du sol, en particulier pour les racines de ligneux (Chêne Kermès, Pin d'Alep, ...). AbDalla suppose alors que cet épaississement entraîne une diminution de la contrainte au niveau de l'apex qui peut alors vaincre cette nouvelle contrainte axiale plus faible. Le méristème s'allonge à nouveau jusqu'à ce que la racine atteigne une zone dans laquelle la pression inhibe l'élongation axiale. Le processus décrit précédemment est repris et la racine continue de se développer avec une section épaissie. Des conclusions analogues ont été tirées par Goss (1974) qui, par des observations plus fines, remarque que l'augmentation de diamètre de la racine est due à l'augmentation de la largeur des cellules du cortex.

L'ensemble de ces travaux est, semble-t-il, réalisé dans des conditions parfois critiquables; nous avons précédemment signalé que dans le cas où c'est la force développée par la racine (GILL \& BOLT, 1955 ; TAYLOR \& RATLIFF, 1969) qui est mesurée, l'environnement de la racine peut poser problème. De plus, il peut se produire une déformation de la racine. Dans les autres essais, où la pression de croissance racinaire est assimilée à la pression extérieure maximum que peut supporter la racine sans présenter de modification, il est possible que la valeur limite de pression ne corresponde pas rigoureusement à la réaction du milieu à la croissance racinaire, mais soit aussi fonction de l'élasticité du milieu. Par ailleurs, il est regrettable que des mesures d'allongement de racines n'accompagnent pas toujours ces expériences.

Pour tenter de remédier, tout au moins en partie à ces inconvénients, nous avons réalisé en laboratoire un dispositif permettant de déterminer à la fois la force et l'allongement de la racine. Pour mettre en relations rigoureuses les déformations possibles enregistrées par les structures végétales racinaires avec la pression exercée par la racine au point d'impact, il est alors indispensable de placer directement la racine en contact avec un obstacle mécanique dont l'aptitude à la flexion est variable et sur lequel la force exercée est mesurée. Ces mesures constituent en quelque sorte un test destiné à connaître le sens de variation des principaux facteurs mis en jeu.

\section{MATÉRIEL ET MÉTHODES}

Le dispositif imaginé et réalisé au laboratoire permet de mesurer la force exercée par la racine séminale en croissance face à un obstacle élastique.

Le système de mesure se compose (fig. 2, photo 1) d'une lamelle d'acier montée en poutre posée sur 2 appuis simples. Une jauge extensométrique collée en son milieu enregistre les déformations relatives de la lamelle (on suppose en effet que l'épaisseur de la jauge est négligeable et que ses déformations sont celles de la lamelle provoquées par la racine en croissance. Ces déformations $\frac{\Delta a}{a}$ sont exprimées en $\mu \mathrm{m} / \mathrm{m}$ et appelées $\mathcal{E}$.

Pour une lamelle dont les caractéristiques géométri- 


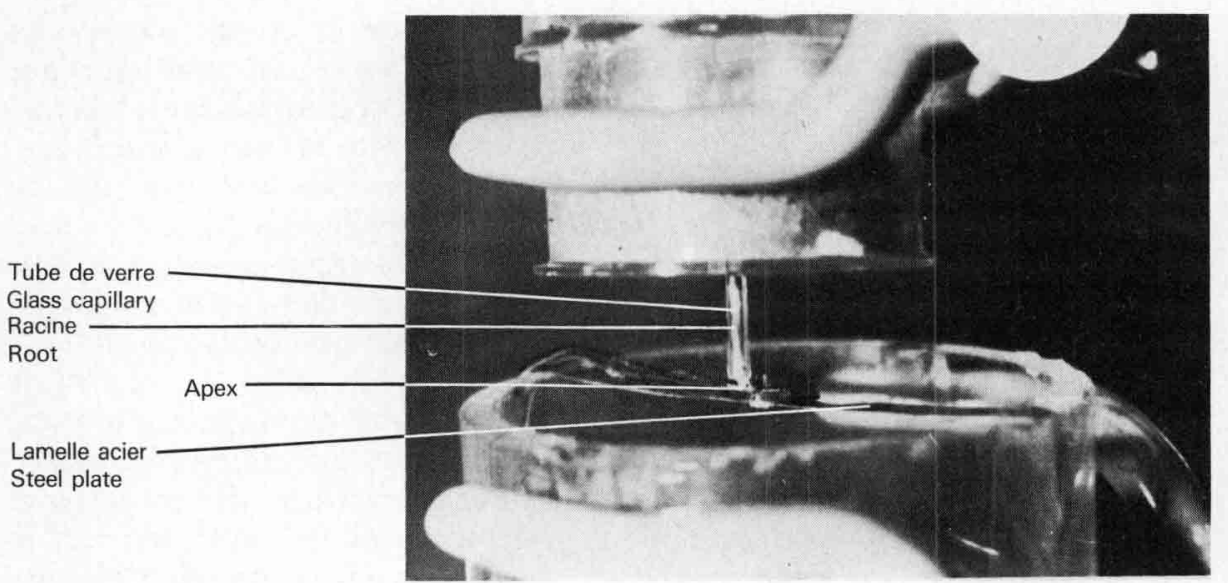

Photo I

Dispositif expérimental avec une racine. Experimental apparatus with root.

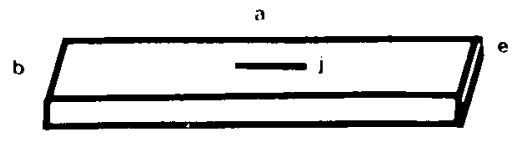

a,b.e : dimensions de la lamelle

j: jauge extensométrique

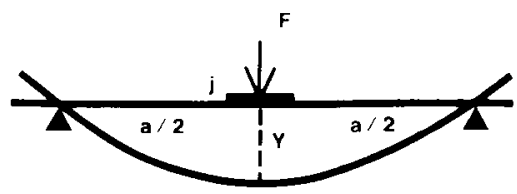

Sous l'action d'une charge $F$

la lamelle prend une fleche $Y$

Figure 2

Schéma théorique de la lamelle montée en poutre avec deux appuis simples:

$a$ : portée ; $b$ : largeur ; $c$ : épaisseur.

Schematic diagram of a beam with two simple supports; $a: \operatorname{span} ; b:$ width $; c:$ thickness.

ques, exprimées en $\mathrm{m}$, sont $\mathrm{a}:$ la portée, $\mathrm{b}:$ la largeur et e : l'épaisseur, le calcul théorique de la résistance des matériaux conduit à une relation entre $\mathrm{F}$ la force appliquée (au milieu de la lamelle) et Y la flèche prise par la lamelle (déplacement du centre de la lamelle) (fig. 2).

$$
\mathrm{F}=\frac{48 \cdot \mathrm{EI}}{\mathrm{a}^{3}} \cdot \mathrm{Y}
$$

avec $\mathrm{E}=$ module d'élasticité de l'acier (module de YOUNG) exprimé en Pascal $\left(\mathrm{N} / \mathrm{m}^{2}\right)$

I = moment d'inertie de la lamelle calculé à partir de la dimension ayant la même direction que l'effort $\mathrm{F}\left(\mathrm{I}=\frac{\mathrm{b} \mathrm{e}^{3}}{12}\right)$.

Nous appelons $\mathrm{K}=\frac{48 \mathrm{EI}}{\mathrm{a}^{3}}=\frac{4 \mathrm{E} \cdot \mathrm{b} \cdot \mathrm{e}^{3}}{\mathrm{a}^{3}}$ coefficient de raideur de la lamelle exprimé en $\mathrm{N} / \mathrm{m}$. Des dimensions différentes de la lamelle permettent donc d'obtenir des obstacles de raideur différente.

Par ailleurs, la déformation de la lamelle s'exprime par :

$$
\varepsilon=\frac{\mathrm{e} \cdot \mathrm{a}}{8 \mathrm{E} \cdot \mathrm{I}} \cdot \mathrm{F}=\frac{3}{2} \frac{\mathrm{a}}{\mathrm{Eb} \mathrm{e}^{2}} \cdot \mathrm{F}
$$

ou

avec

$$
\begin{aligned}
\mathrm{F} & =\frac{2}{3} \frac{\mathrm{Eb} \mathrm{e}^{2}}{\mathrm{a}} \cdot \varepsilon=\alpha \cdot \varepsilon \\
\alpha & =\frac{2}{3} \frac{\mathrm{Eb} \mathrm{e}^{2}}{\mathrm{a}} .
\end{aligned}
$$

L'étalonnage de la lamelle fournit le coefficient reliant $\mathrm{F}$ à $\varepsilon$ ainsi que la valeur de $\mathrm{K}$ puisque :

$$
K=6 \alpha \cdot \frac{\mathrm{e}}{\mathrm{a}^{2}} .
$$

Des équations (1) et (3), il vient :

$$
\mathrm{Y}=\frac{\alpha}{\mathrm{K}} \cdot \varepsilon \quad \text { ou } \quad \mathrm{Y}=\frac{\mathrm{a}^{2}}{6 \mathrm{e}} \cdot \varepsilon .
$$

La flèche Y prise par la poutre n'est autre que l'allongement axial de la racine.

La mesure proprement dite se déroule de la façon suivante :

- Le dispositif est placé dans une enceinte climatique saturée en eau dont la température est constante et les périodes jour et nuit sont adaptées à la saison.

- Pour des raisons pratiques, nous avons choisi la racine séminale du maïs. Les graines de maïs sont mises à germer sur un dispositif présenté sur la figure 3 . Dès que la racine a atteint une longueur de 5 à $25 \mathrm{~mm}$ environ, la graine est enfermée dans une boîte de sable humide percée à sa base de façon à laisser passer la racine séminale introduite dans un tube de verre lui servant de guide et de frette. L'apex de la racine est ensuite placé au centre de la lamelle de sorte qu'il la touche sans appuyer (photo 1). Au cours du temps, sont enregistrées dans un système d'acquisition de données, les déformations relatives $\varepsilon$ de la jauge, donc de la lamelle. La relation (3) permet de transformer l'ordonnée $\varepsilon$ en

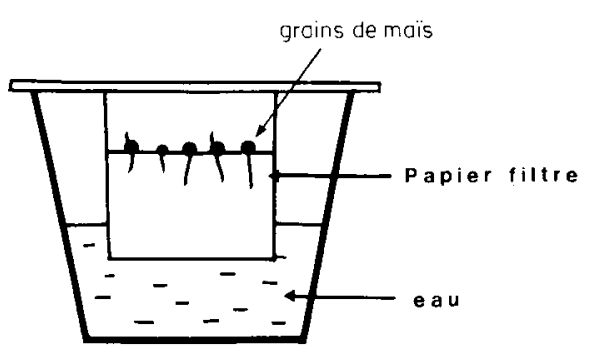

Figure 3

Dispositif utilisé pour la germination des graines de maïs Device used for seed germination. 
force et la relation (5) transforme $\varepsilon$ en $Y$, c'est-à-dire en allongement $\Delta \mathrm{L}$. Nous pouvons alors tracer la courbe représentant l'allongement de chaque racine en fonction du temps (fig. 4).

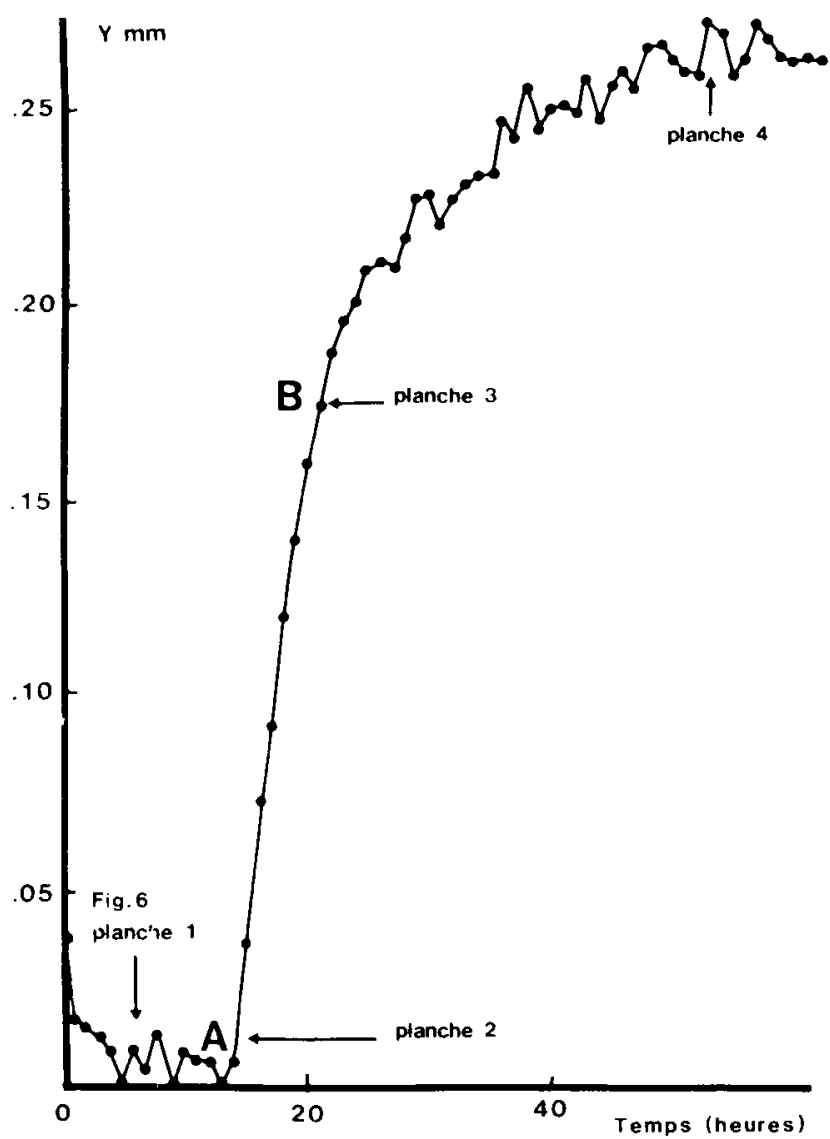

Figure 4

Allongement d'une racine ( $\Delta L)$, face à un obstacle, en fonction du temps.

Root elongation ( $\Delta L$ in $\mu \mathrm{m}$ ) on against obstacle, with time (in $h$ ).

\section{EXPRESSION DES RÉSULTATS}

Cette courbe présente dans tous les cas étudiés trois zones distinctes :

$-\mathrm{t}<\mathrm{t}_{\mathrm{A}}$ : une variation faible et irrégulière de $\Delta \mathrm{L}$ correspondant à la mise au contact de l'apex avec la lamelle élastique ;

$-\mathrm{t}_{\mathrm{A}}<\mathrm{t}<\mathrm{t}_{\mathrm{B}}$ : croissance continue de $\Delta \mathrm{L}$. La racine exerce une force constamment croissante sur la lamelle. La variation de la force ou de la longueur apparaît approximativement linéaire en fonction du temps. Pendant cette phase, l'allongement a lieu sans autre modification perceptible macroscopiquement de la morphologie de la racine (diamètre, orientation). La force mesurée peut donc être assimilée à une force de croissance axiale. La qualité de l'approximation linéaire retenue pour la relation $\Delta \mathrm{L}(\mathrm{t})$ et $\mathrm{F}(\mathrm{t})$ dans cette zone est montrée par les résultats du tableau 1 où sont présentés, pour une dizaine de cas couvrant l'essentiel de la variabilité des courbes obtenues, les coefficients de détermination $\mathrm{r}^{2}$ des régressions calculées. En fait, la validité de cette approximation dépend du choix des limites A et B. Quant au point A, l'incertitude de locali-
TABLEAU 1

Exemples de valeurs des coefficients de corrélation linéaire de la partie $A B$ des courbes expérimentales obtenues pour différentes racines.

Linear regression on the $A B$ experimental curve: values of correlation coefficients.

\begin{tabular}{cc}
\hline \hline $\begin{array}{c}\text { Nombre de points } \\
\text { expérimentaux }\end{array}$ & $r^{2}$ \\
\hline 36 & 0,970 \\
27 & 0,975 \\
24 & 0,983 \\
18 & 0,999 \\
36 & 0,980 \\
60 & 0,993 \\
48 & 0,986 \\
36 & 0,984 \\
24 & 0,985 \\
30 & 0,979 \\
30 & 0,981 \\
\hline
\end{tabular}

sation est faible, le changement d'allure des courbes étant dans tous les cas aussi rapide que le montre la figure 4. En revanche, la variation progressive de la pente implique une incertitude beaucoup plus grande sur la position du point B, au moins dans certains cas, dont celui présenté. Toutefois, la faible variation de la pente implique également un effet réduit du choix du point B sur les paramètres de l'ajustement linéaire. On peut donc dans cette zone, à partir de l'étalonnage préalable de la lamelle et de la variation $\left(\varepsilon_{B}-\varepsilon_{A}\right)$, calculer la force $\mathrm{F}$, l'allongement $\Delta \mathrm{L}$ et la vitesse d'élongation $\frac{\Delta \mathrm{L}}{\Delta \mathrm{t}}$

$-\mathrm{t}>\mathrm{t}_{\mathrm{B}}$ : la croissance est perturbée et des modifications morphologiques apparaissent : épaississement au-dessus du méristème, changement de direction de l'apex qui tend à contourner l'obstacle. L'allure de la courbe au-delà de $B$ peut être différente de celle présentée à la figure 4, ce qui empêche, en particulier, d'utiliser une forme d'ajustement unique pour traiter statistiquement l'ensemble des courbes. Dans cette zone, l'indication fournie par la jauge extensométrique ne résulte plus du seul allongement axial.

\section{DISCUSSION}

\section{A. Détermination des paramètres de croissance raci- naire}

Les valeurs de la force de croissance calculées comme nous venons de le décrire sont, dans l'ensemble, du même ordre de grandeur que celles obtenues par d'autres auteurs: GILL \& MILLER, 1956 ; ABDALlA et al., 1969 ; WhITELEY et al., 1981. Dans les conditions de nos expériences, c'est la réaction de l'obstacle à la force fournie par toute la zone d'élongation racinaire, que nous mesurons. Cette réaction dépend de la raideur $\mathrm{K}$ de l'obstacle (nous l'exposerons dans le prochain article) : elle est de l'ordre de 0,01 à $0,25 \mathrm{~N}$ correspondant, pour une racine de $1 \mathrm{~mm}$ de diamètre, à des pressions de 10 à $250 \mathrm{KPa}$.

Parallèlement, les vitesses de croissance se situent 
entre 0,05 et $0,8 \mathrm{~mm} / \mathrm{h}$. Il faut bien insister sur le fait que ces valeurs expérimentales de force et de vitesse sont relatives à l'allongement axial de la racine avant toute déformation dans des conditions opératoires bien précises. Ces conditions peuvent être sujettes à critiques sur deux points :

- le rapport du diamètre du tube guide à celui de la racine a une importance. En effet, s'il est grand, l'angle fait par la racine avec la surface de la lamelle peut varier et modifier la valeur de la force atteinte au point B de la courbe de la figure 4 (DEXTER, 1978). S'il est petit, par contre, la racine peut subir des contraintes latérales non maîtrisables et être gênée dans son développement ;

- la plantule ne vit que sur les réserves de la graine et l'alimentation hydrique n'est assurée que par le sable contenu dans la boîte. Cependant, l'atmosphère de l'enceinte est saturée et nous n'avons jamais observé de plasmolyse des tissus pendant la durée de l'expérience (quelques heures).

\section{B. Evolution comparée des structures cellulaires de l'apex de la racine}

L'étude de la morphologie cellulaire en relation avec les contraintes mécaniques exercées paraît apporter quelques indications intéressantes. Nous avons comparé, à l'aide de coupes cytologiques, l'évolution des structures épidermiques d'une racine en croissance sur la lamelle (FÉRAUD-VIARDOT, 1985).

Les coupes longitudinales sont rassemblées sur la figure 6. La comparaison des planches 2-3-4 avec la planche 1 (racine témoin), présentant une coiffe nette et pointue et un centre quiescent bien distinct du méristème, est très intéressante (fig. 5). La coiffe de la racine (planche 2 ) est moins pointue, les cellules régénératrices sont plus compactes et il y a beaucoup plus de cellules de desquamation. Sur la planche 3 , nous observons une diminution de la zone méristématique tandis que les cellules de la zone d'élongation sont bien distinctes : ce phénomène s'accentue dans le cas de la

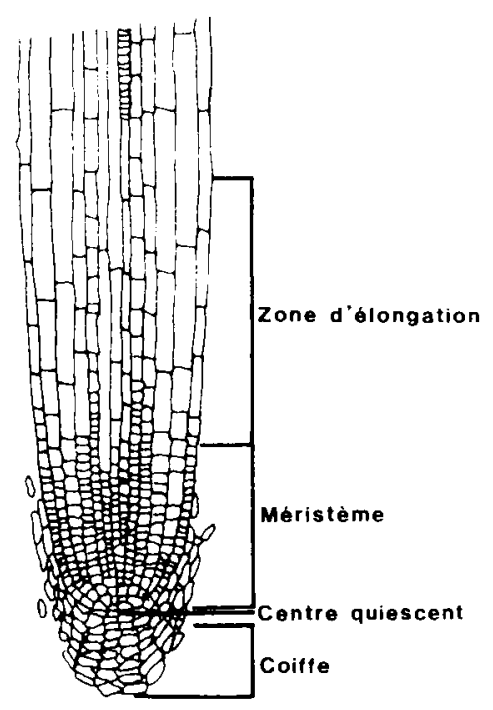

Figure 5

Schéma simplifié de la zone de croissance d'une racine : coupe longitudinale.

Simplified diagram of the growing zone of a root: longitudinal section. racine ayant donné son maximum de force sur l'obstacle : planche 4. Des coupes transversales de racines en croissance sur l'obstacle laissent supposer, mais ce résultat est à vérifier dans les essais prochains, que les mitoses de la zone méristématique diminuent dès que la racine heurte l'obstacle ; il semble même qu'il y a arrêt des mitoses quand la racine appuie sur l'obstacle. L'allongement axial, résultant en conditions non limitantes des phénomènes de multiplication et d'élongations cellulaires, ne serait dû qu'à l'allongement des cellules de la zone d'élongation quand la racine se trouve sur un obstacle. Donc avec la méthode expérimentale présentée, ce serait la réaction de la lamelle à la force développée par la seule zone d'élongation que nous mesurerions.

\section{CONCLUSION}

La revue bibliographique faite en début de texte met en évidence les difficultés à mener à bien une étude quantitative des effets d'une contrainte mécanique sur la croissance racinaire. Dans le sol, il n'y a pas de possibilité d'estimation de contrainte mécanique exacte affectant la croissance d'une racine. La méthode expérimentale ici décrite, permet de tester assez facilement les racines quant à leur comportement mécanique visà-vis d'un obstacle à leur croissance, si les conditions hydriques et nutritives sont favorables. Nous sommes capables alors de déterminer simultanément la force et la vitesse de croissance axiale d'une racine. Parallèlement, les quelques observations cytologiques réalisées laissent entrevoir l'existence de corrélation avec les paramètres mécaniques de l'environnement. C'est pourquoi il paraît intéressant de poursuivre ce travail en faisant varier le coefficient de raideur $K$ et en prenant en compte les caractéristiques de la racine (longueur, diamètre, poids de la graine, ...). Ainsi, nous pourrons connaître d'une part la variation de la force et de la vitesse de croissance, d'autre part l'évolution des structures épidermiques de l'apex de la racine en fonction des paramètres liés au végétal et au milieu environnant.

Reçu le 24 mars 1986 Accepté le 7 mai 1987.

\section{REMERCIEMENTS}

Nous remerçions Monsieur le Professeur Espagnac, Professeur de Biologie végétale à la Faculté des Sciences d'Avignon, d'avoir bien voulu accueillir dans son laboratoire Laurence FéraudVIARDOT (Stagiaire I.U.T.) pour la réalisation et l'interprétation des coupes cytologiques.

Figure 6

Coupes longitudinales.

Longitudinal sections.

Planche 1: Racine témoin avant contact avec l'obstacle $(G \times 10)$. Reference root which is not in contact with the obstacle $(G \times 10)$.

Planche 2 : Racine commençant à appuyer sur l'obstacle. Root at the beginning of its growth on the obstacle.

Planche 3 : Racine appuyant sur un obstacle. Root growing on the obstacle.

Planche 4 : Racine ayant développé une force maximale sur l'obstacle. Root which has given the greatest force against the obstacle. 

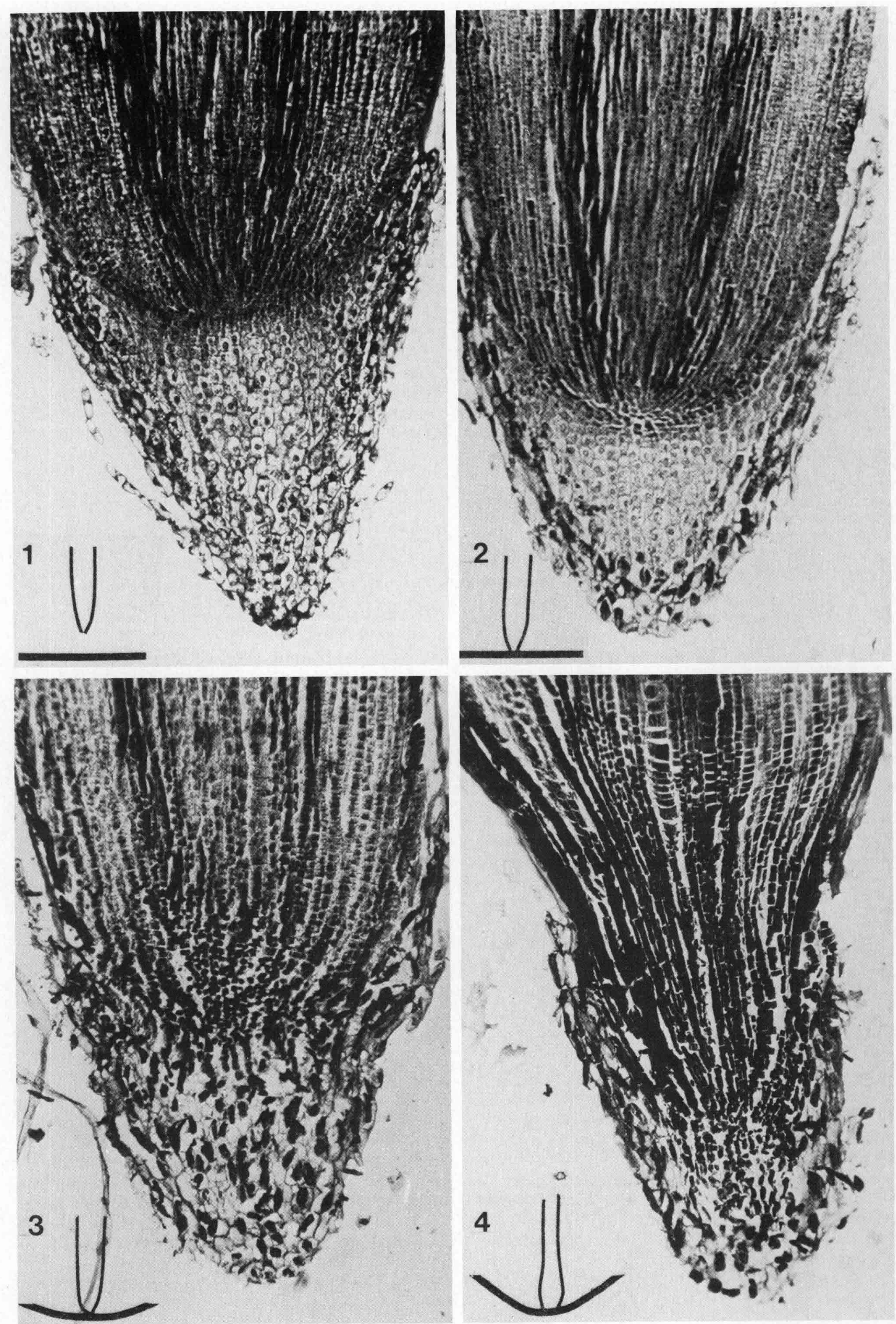


\section{RÉFERENCES BIBLIOGRAPHIQUES}

Abdalla A. M., Hettiaratchi D. R. P., Reece A. R., 1969. The mechanics of root growth in granular media. J. agric. Eng. Res., 114 (3), 236-248.

Aubertin G. M., Kardos L. T., 1965. Root growth through porous media under controlled conditions. I. Effect of pore size and rigidity. Soil Sci. Soc. Proc., 29 (3), 290-293.

Barley K. P., 1962. The effects of mechanical stress on the growth of roots. J. exp. Bot., 13 (37), 95-110.

Barley K. P., Greacen E. L., 1967. Mechanical resistance as a soil factor influencing the growth of roots and underground shoots. Adv. Agron., 19, 1-43.

Batchelder A. R., Bouldin D. R., 1972. Technique of determination root elongation through soil layers of different physical and chemical properties. Agron. J., 64, 49-52.

Boehle J., Mitchell W. H., Kresge C. B., Kardos L. T., 1963. Apparatus for taking soil root cores. Agron. J., 55, 208-209.

Dexter A. R., 1978. A stochastic model for the growth of roots in tilled soil. J. Soil Sci., 29, 102-116.

Dexter A. R., Hewitt J. S., 1978. The deflection of plant roots. $J$. agric. Eng. Res., 23, 17-22.

Drew M. C., 1975. Comparison of the effects of a localized supply of phosphate, nitrate, ammonium and potassium on the growth of the seminal root system and the shoot in barley. New Phytol., 75, 479-490.

Eavis B. W., 1967. Mechanical impedance and root growth. Proc. Agr. Eng. Symp. (Silsoe England).

Eavis B. W., Ratliff L. F., Taylor H. M., 1969. Use of a dead-load technique to determine axial root growth pressure. Agron. J., 61, 640-643.

Féraud-Viardot L., 1985. Croissance racinaire. Cytologie de la racine séminale de maïs en croissance sur un obstacle déformable et non pénétrable. Rapport de stage, IUT Biologie appliquée, Montpellier.

Gardner W. R., 1964. Relation of root distribution of water uptake and availability. Agron. J., 56, 41-45.

Gill W. R., Bolt G. H., 1955. Pfeffer's studies of the root growth pressures exerted by plants. Agron. J., 47, 166-168.

Gill W. R., Miller R. D., 1956. A method for study of the influence of mechanical impedance and aeration on the growth of seedling roots. Soil Sci. Soc. Proc., 20 (2), 154-157.

Gooderham P. T., 1973. Soil physical conditions and plant growth. PhD Thesis, Univ. of Reading, $150 \mathrm{p}$.

Goss M. J., 1974. Effect of mechanical impedance on root growth. PhD Thesis, Univ. of Reading, $135 \mathrm{p}$.

Melhuish F. M., 1968. A precise technique for measurement of roots and root distribution in soils. Ann. Bot., 32, 15-22.

Nguyen P. T., 1977. Mechanics of soil deformations in relation to root growth. PhD Thesis, University of Newcastle, $112 \mathrm{p}$.
Nye P. H., Marriott F. C. H., 1969. A theoretical study of the distribution of substances around root resulting from simultaneous diffusion and mass flow. Plant Soil, 30, 459-478.

Périgaud S., 1966. Effet de la résistance mécanique et du déficit en oxygène sur la croissance du maïs. C. R. Acad. agric., 695-707.

Périgaud S., 1966. Effet du manque d'oxygène et de l'excès d'eau au niveau des racines sur la croissance et la nutrition globale de la plante. Ann. agron., 18, 485-506.

Pfeffer W., 1893. Druck und Arbeitsleistung durch wachsende Pflanzen. Abhandhungen der Königlich Sächsischengesellschaft der Wissenschaften, 33, 235-474.

Phillips R. E., Dom Kirkham, 1962. Mechanical impedance and corn seedling root growth. Soil Sci. Soc. Am. Proc., 26, 319-322.

Prebble R. E., 1970. Root penetration of smeared soil surfaces. Exp. Agric., 6, 303-308.

Reicosky D. C., Millington R. J., Peters D. B., 1970. A comparison of three methods for estimating root length. Agron. J., 62, 451-453.

Russell R. S., Goss M. J., 1974. Physical aspects of soil fertility. The response of roots to mechanical impedance. Neth. J. Agric. Sci., 22, 305-318.

Stolzy L. H., Barley K. P., 1968. Mechanical resistance encountered by roots entering compact soils. Soil Sci., 105-5, 297.

Tardieu F., Manichon H., 1986. Caractérisation en tant que capteur d'eau de l'enracinement du maïs en parcelle cultivée. I. Discussion des critères d'étude. Agronomie, 6 (4), 345-354.

Tardieu F., Manichon H., 1986. Caractérisation en tant que capteur d'eau de l'enracinement du maïs en parcelle cultivée. II. Une méthode d'étude de la répartition verticale et horizontale des racines. Agronomie, 6 (5), 415-425.

Taylor H. M., Burnett E., 1964. Influence of soil strength on the root growth habits of plants. Soil Sci., 98, 174-180.

Taylor H. M., Ratliff L. F., 1969. Root elongation rates of cotton and peanuts as a function of soil strength and soil water content. Soil Sci., 108 (2), 113-119.

Taylor H. M., Ratliff L. F., 1969. Root growth pressures of cotton, peas and peanuts. Agron. J., 61, 398-403.

Whiteley G. M., Dexter A. R., 1983. Behaviour of roots in cracks between soil peds. Plant Soil, 74 (2), 153-162.

Whiteley G. M., Dexter A. R., 1984. The behaviour of roots encountering cracks in soil. I. Experimental methods and results. Plant Soil, 77, 141-149.

Whiteley G. M., Utomo W. H., Dexter A. R., 1981. A comparison of penetrometer pressures and the pressures exerted by roots. Plant Soil, 61, 351-364.

Whiteley G. M., Hewitt J. S., Dexter A. R., 1982. The buckling of plant roots. Physiol. Plant, 54, 333-342. 